\title{
Regards
}

\section{L'expérimentation animale : une responsabilité à dire et à partager}

\author{
Évelyne Lhoste ${ }^{1}$, Béatrice de Montera ${ }^{2}$ \\ 1 Physiologiste et responsable du service de communication, Inra, Unité déconcentrée d'appui à la recherche, 78350 Jouy-en-Josas, \\ France \\ 2 Biologiste et philosophe, Inra, Unité de recherche Biologie du développement et reproduction, 78350 Jouy-en-Josas, France
}

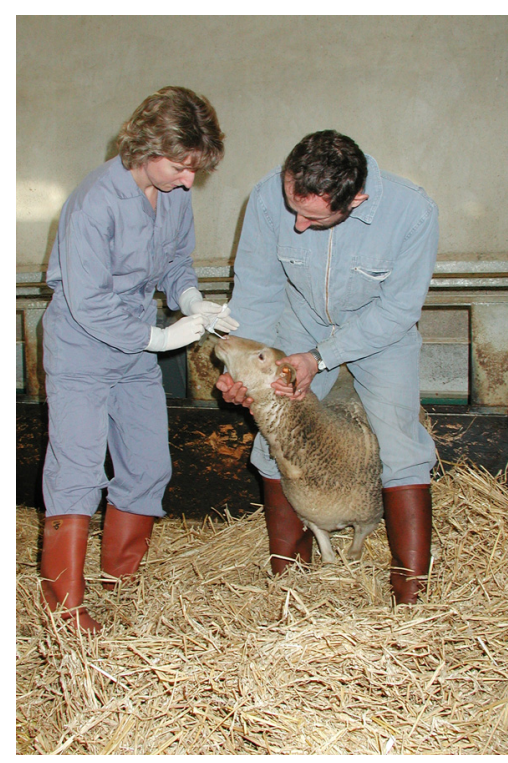

Écouvillonnage nasal chez le mouton pour le suivi de la brucellose. (c) INRA-Alain Beguey.

\section{Introduction}

La communauté scientifique a, vis-à-vis de l'expérimentation animale, une position qui est loin d'être unanime. Si la plupart des physiologistes la jugent «nécessaire et indispensable », d'autres biologistes sont plus critiques, voire y sont opposés. Dans notre propre centre de recherche (Inra, Jouy-en-Josas), une enquête effectuée auprès de 192 agents a révélé que $9 \%$ des participants sont opposés à l'utilisation des animaux à des fins d'acquisition de connaissance, alors que $95 \%$ d'entre eux sont d'accord lorsque l'objectif des recherches est d'améliorer

Auteur correspondant : E. Lhoste, evelyne.lhoste@jouy.inra.fr la santé humaine (Grohs et Bernard, 2007). Nous relatons ici une expérience conduite depuis plus de 4 ans au sein de ce centre. Celle-ci a consisté à mettre en place une démarche réflexive sur la pratique de l'expérimentation animale, par le biais d'une formation à l'éthique ouverte à tout le personnel. Elle a permis notamment d'aider des acteurs du collectif de recherche, les animaliers, à mettre des mots sur les conflits de valeurs qui surviennent au cours de leur pratique expérimentale. L'objectif de cet article est d'attirer l'attention sur le fait que ces conflits de valeurs doivent être reconnus et pris en compte. Or ils ne peuvent pas toujours être résolus par les règles éthiques implicitement admises par la réglementation européenne. Par conséquent, il nous semble 
important que les questions éthiques soulevées par l'utilisation des animaux en recherche soient discutées au sein des équipes et que chacun en porte la responsabilité.

\section{Éléments de contexte}

Plus de la moitié des équipes du centre Inra de Jouy-en-Josas sont impliquées, de près ou de loin, dans des protocoles utilisant des animaux. De plus, le centre tire sa spécificité de la diversité et de l'originalité des modèles animaux qui y sont développés. Ainsi, sur les 1200 personnes qui travaillent à Jouy, 70 sont des animaliers. Les unités expérimentales hébergent annuellement 24000 rongeurs et lagomorphes, 2500 gros mammifères et 12500 poissons.

Depuis plusieurs années, la question de l'expérimentation animale fait l'objet d'une démarche réflexive relayée auprès du personnel. Des formations ouvertes à tous sont organisées régulièrement avec pour objectif de s'interroger sur l'utilisation des animaux en recherche. Les thématiques abordées vont d'un cours introductif à l'éthique à des conférences sur des questions liées aux biotechnologies animales. Le nombre et le statut professionnel des participants sont variables et dépendent des thématiques abordées. La visibilité de ces formations est accrue par une diffusion plus large à travers le bulletin d'information du centre. Ces diverses actions sont pilotées par un groupe de réflexion sur l'éthique en expérimentation animale - GREEA-Jouy ${ }^{1}$. L'objectif est de sensibiliser le personnel à la démarche éthique afin que chacun puisse exercer sa responsabilité dans ce domaine et être informé des risques liés aux éventuels conflits moraux auxquels peut être confronté le personnel en contact direct avec les animaux.

Les conclusions présentées dans cet article émanent d'observations et de témoignages recueillis sur plusieurs années au sein du centre : 1) commentaires lors des conférences-débats dans le cadre d'un cycle de formation intitulé "Animaux en recherche : questions et débats »; 2) comptes rendus d'une quinzaine de réunions de travail visant la conception, avec les animaliers, d'un documentaire vidéographique présentant leur métier ${ }^{2}$; 3) une enquête auprès de 17 animaliers, dont l'objectif était de mieux percevoir les valeurs sous-jacentes au métier ; 4) six entretiens en face-à-face avec des animaliers et des chercheurs conduits dans le cadre d'un mémoire de recherche (Lhoste, 2003). L'originalité de cette recherche

\footnotetext{
${ }^{1}$ Les résultats de cette expérience sont diffusés dans le bulletin interne et sur le site web du centre. Ils ont également fait l'objet d'une communication (Lhoste et al., 2008) au $34^{\mathrm{e}}$ colloque de l'Association française des sciences et techniques de l'animal de laboratoire (4-6 juin 2008, Strasbourg).

2 DVD Paroles d'animaliers, Inra, 38 minutes, 2008. Disponible auprès des auteurs.
}

de terrain réside dans le fait qu'elle a été conduite par des chercheurs impliqués dans l'expérimentation animale et que le travail de révélation des valeurs sousjacentes se fait à partir d'un matériau anthropologique. Si nos conclusions concordent avec celles d'autres travaux (Birke et al., 2007), notre approche n'a pas pour autant l'ambition de remplacer une étude sociologique, psychosociologique ou anthropologique.

\section{Plusieurs "éthiques animales » coexistent au sein du collectif de recherche}

Cette affirmation nécessite quelques précisions. D'une part, nous définissons ici l'éthique comme un «savoir-vivre » considérant ce qui est bon pour les individus concernés. Il s'agit donc d'une éthique appliquée qui se préoccupe de cas concrets. Il ne s'agit pas ici de règles de conduite déontologiques, mais d'une réflexion en amont des pratiques, au niveau des valeurs qui président aux décisions et aux actions. D'autre part, nous parlons d'éthiques au pluriel dans la mesure où les membres du collectif de recherche n'accordent pas le même statut moral aux animaux et ne partagent pas la même éthique.

Ce pluralisme vient à l'encontre de l'unicité implicitement admise dans la réglementation européenne relative à la protection des animaux utilisés à des fins expérimentales (directive 86/609/CEE). En effet, celle-ci se fonde sur un utilitarisme dit pathocentrique. Cette doctrine cherche, de manière opératoire, à évaluer l'utilité de l'expérimentation tout en valorisant le fait de ne pas faire souffrir un être sensible. Jeremy Bentham (1789) fut le premier à la faire rentrer dans le périmètre protecteur de la considération morale ${ }^{3}$. Cette conception est basée sur la distinction hobbesienne entre la loi qui nous lie, et le droit qui nous donne une liberté à l'intérieur d'un certain périmètre (Hobbes, 1651, trad. fr. de 1982, p. 245). La conception du droit moral et celle de l'éthique appliquée que nous utiliserons ici sont essentiellement basées sur le fait d'offrir cette protection (de Montera, 2000). Une certaine interprétation de cette éthique est reconnue par la communauté scientifique sous la forme de recommandations pratiques issues d'une interprétation simplifiée de la règle des $3 R$ - Remplacer, Réduire, Raffiner (Russell et Burch, 1959). Ces recommandations sont notamment suivies par les comités d'évaluation éthique des protocoles expérimentaux.

L'utilitarisme est difficile à mettre en œuvre lorsqu'il s'agit de sacrifier les intérêts de certains individus (et parfois même leur vie) au bénéfice du plus grand nombre. La garantie d'une équité de traitement entre les membres de

\footnotetext{
3 «La question n'est pas : peuvent-ils raisonner, ni : peuventils parler, mais : peuvent-ils souffrir? », édition de 1996, p. 283 (traduction des auteurs).
} 
la communauté est alors nécessaire, ce qui ne va pas de soi (de Montera, 2000). D'ailleurs, Peter Singer (1975) luimême, utilitariste et initiateur de la libération animale, reconnaît que cette équité n'exclut pas que certaines vies vaillent plus que d'autres, justifiant l'utilisation des animaux, voire de vies humaines déficientes, au bénéfice de l'humanité.

\section{Typologie des acteurs de l'expérimentation animale}

Tous les membres du collectif de recherche ne se reconnaissent pas dans cette éthique, en raison de la diversité des relations qu'ils entretiennent avec les animaux, d'une part, et de la pluralité des visions qu'ils ont de la science, d'autre part.

Nous avons séparé le collectif de recherche en trois catégories : les concepteurs de protocoles expérimentaux, les réalisateurs de ces protocoles et les animaux. Les deux catégories «humaines » ne correspondent pas rigoureusement à la classification habituelle en chercheurs, techniciens et animaliers. Elles se distinguent principalement par le degré de responsabilité vis-à-vis des protocoles expérimentaux et la proximité physique avec les animaux.

Les concepteurs de protocoles (des chercheurs) assument la responsabilité scientifique des projets de recherche et en pilotent la réalisation. Du fait de leur éloignement géographique ou disciplinaire, la plupart d'entre eux se limite à exploiter des données ou des échantillons recueillis sur les animaux par les techniciens ou les animaliers. Toutefois, certains techniciens ne font que traiter des échantillons sans participer à leur recueil, alors que des chercheurs «à la paillasse » interviennent «sur » les animaux pour des prélèvements d'échantillons, des actes chirurgicaux ou au moment de l'euthanasie. Seuls les animaliers ont pour mission de prendre soin des animaux en dehors de la période d'expérimentation proprement dite. Il leur arrive notamment de leur donner naissance, ce qui n'est pas sans conséquence pour la suite de leur relation à l'animal.

Les animaliers ont retenu toute notre attention parce qu'ils sont indispensables à la recherche sur l'animal alors même que l'importance de leur rôle est souvent négligée. Ils se perçoivent eux-mêmes comme "un maillon » de la chaîne qui représente le travail de recherche, ce dont ils sont fiers : «sans animaliers, pas d'animaux et sans animaux, pas de recherche ${ }^{4} »$. Ils participent tous aux expérimentations (prélèvements d'échantillons, récolte de données) tout en prenant soin des animaux au quotidien, ce qui leur confère une double identité et un rôle de médiation : ils traduisent les comportements des animaux

\footnotetext{
${ }^{4}$ Les mots d'animaliers sont extraits des entretiens en face-àface ou du documentaire Paroles d'animaliers. Dans le deuxième cas, une note de bas de page le précise à la suite de la citation.
}

en information pour les chercheurs. Leur double identité transparaît, par exemple, dans leur attachement à des valeurs typiques des deux mondes. Elle est claire chez les animaliers «gros animaux » qui s'identifient au travers de valeurs comme l'autonomie, la rigueur et l'efficacité. Si la rigueur est une valeur typique de la recherche, l'efficacité est, quant à elle, propre aux professionnels de l'élevage (Dockès et Kling-Eveillard, 2007).

On distingue deux populations d'animaliers selon qu'ils s'occupent des animaux de laboratoire « conventionnels » (rongeurs) ou des animaux domestiques (bovins, ovins, porcins et caprins). Les premiers ont reçu une formation adaptée à l'expérimentation animale et, de manière générale, ils sont intéressés par le côté technique. Au contraire, ceux qui s'occupent des gros animaux ont reçu une formation en élevage et sont souvent issus du monde agricole. Certains d'entre eux sont d'anciens exploitants, d'autres des jeunes en attente de pouvoir reprendre la ferme familiale. C'est à leur entrée à l'Inra qu'il leur a été demandé de participer à des études expérimentales, ce qui représente une activité intéressante et valorisante pour la plupart. Ils acquièrent, sur place et le plus souvent par compagnonnage, des savoir-faire spécifiques.

«J'ai atterri à l'Inra à la fin de mon service militaire. . p par la France agricole. Je suis issu du milieu agricole. J'ai fait toutes mes études dans ce milieu. [... ] J'ai passé le concours Inra [...] » (animalier bovins); "Mes parents sont agriculteurs dans le Doubs [...] D'ici une dizaine d'années, peut-être que je quitterai l'Inra pour reprendre l'exploitation familiale. » (Animalier bovins ${ }^{5}$.)

Même si leur marge de manœuvre semble plutôt restreinte, c'est aussi des animaux que va dépendre le résultat final de la recherche. Il s'agit de mammifères dans notre étude. Selon les espèces considérées, ils diffèrent par leurs capacités cognitives et la richesse de leur univers mental. Les conditions dans lesquelles ils vivent sont régies par la loi, qui leur reconnaît le statut d'être sensible. Mais chaque être humain (et donc chaque expérimentateur) est plus ou moins sensible au sort réservé aux animaux, suivant son histoire personnelle et sa culture. Ainsi, le statut des animaux varie en fonction des espèces et de la sensibilité envers ces espèces. En particulier, la valeur symbolique des bovins est supérieure à celle des autres animaux domestiques, lesquels ont un statut différent selon que la personne en contact est d'origine rurale ou urbaine. Par exemple, le lapin est considéré par certains comme un animal de compagnie, alors que d'autres y voient surtout une denrée alimentaire. Quant aux rongeurs, ils bénéficient en général de peu de considération et, pour nos animaliers "gros animaux », ce sont des « vermines nuisibles » dont ils ne « se voient pas s'occuper».

Le statut de l'animal va également dépendre de la culture de l'organisme de recherche et de l'évolution

${ }^{5}$ Extraits de Paroles d'animaliers 
de cette culture à travers les avancées technologiques. À l'Inra, on distingue les espèces de laboratoire (rats et souris) de celles d'élevage (poissons, lapins, chèvres, bovins...). Les représentations des espèces animales ont néanmoins changé puisqu'en 1950, le centre de Jouy-enJosas était dédié à la zootechnie, discipline dont le but était de rationaliser la production des animaux d'élevage, et qu'aujourd'hui, l'expression «biotechnologies animales » résonne dans la société comme un ensemble de pratiques entraînant une réification accrue de l'animal en tant qu'objet biotechnique. Mais cette apparente instrumentalisation du vivant coexiste avec la vision des éthologues qui prennent en compte l'existence d'émotions chez les animaux d'élevage (Despret et Porcher, 2007). Les clones de bovins ont fait l'objet d'études de comportement bien que les animaliers considèrent qu'une fois adultes, "ce sont des animaux comme les autres ».

\section{La relation à l'animal au cœur de l'éthique}

Nous soutenons l'hypothèse que la relation à l'animal est au cœur de l'éthique de chacun des membres du collectif.

\section{Le défi du contact direct avec les animaux}

L'éthique des chercheurs, telle qu'analysée par Raphaël Larrère (2002), diffère de l'éthique utilitariste. Partagés entre leur mission sociale (produire des connaissances) et la reconnaissance aux animaux du statut d'être sensible, ils vont opérer un «bricolage éthique » en articulant un utilitarisme pathocentrique avec une certaine interprétation de la déontologie kantienne qui légitime l'instrumentalisation des animaux en la transformant en une question de responsabilité de l'homme vis-à-vis de lui-même $^{6}$ (Kant, 1924, trad. fr. de 1997, p.p. 391-3937). Tout en présentant l'avantage d'une évaluation d'apparence rationnelle (le «calcul coût-bénéfice » issu d'une éthique utilitariste), cette éthique spontanée ne permet théoriquement pas d'intégrer les animaux dans le périmètre moral, alors même que la science a démontré que les animaux étaient des êtres sensibles.

\footnotetext{
${ }^{6}$ Cette éthique exclut les animaux de la communauté morale et ne leur reconnaît aucun droit. Kant pose comme précepte que tout acte de cruauté envers les animaux est répréhensible uniquement parce qu'il est moralement dégradant pour l'homme. Dans la philosophie de Kant en effet, l'homme est le seul qui ne doive jamais être considéré seulement comme un moyen. Les chercheurs s'arrangent avec cet impératif et, dans le cadre de la recherche biomédicale, les molécules sont d'abord testées sur les animaux, puis sur l'homme.

7 Ouvrage établi par Paul Menzer en 1924, sur la base de trois manuscrits d'élèves de Kant qui avaient suivi ses cours d'éthique entre 1775 et 1780 .
}

Cependant, ce bricolage ne résiste pas bien à une confrontation physique avec la douleur animale. C'est peut-être pourquoi certains chercheurs préfèrent rester à distance. Les témoignages que nous avons recueillis démontrent bien que ceux qui ont eu l'expérience d'une relation avec l'animal lui accordent spontanément un statut d'être sensible, tout en acceptant l'idée que la relation historique entre l'homme et l'animal implique la souffrance et la mort pour ce dernier. «L'anesthésie à l'éther, ça m'a toujours dérangée... Je ne me suis jamais habituée. Le fait de voir que l'animal devait souffrir, ça m'a toujours gênée. » (Chercheure rongeurs.)

Les chercheurs loin de l'expérimentation ou les étudiants inexpérimentés, qui acceptent l'expérimentation animale sans relever le défi du contact direct avec les animaux, perçoivent le bien-être de ces derniers comme une valeur abstraite.

Le problème inhérent aux méthodes et aux instruments de mesure qui tendent à éloigner le chercheur de l'objet de son étude est pourtant un élément à prendre en compte. À travers le procédé expérimental, le chercheur peut être enclin à suivre la perspective d'une idéologie telle que définie par Georges Canguilhem (1977) liée à la perte de la distance critique nécessaire à l'objet d'étude et qui peut alors lui faire croire qu'un rat dans un labyrinthe, par exemple, va agir naturellement alors qu'il répond en fait le plus souvent à ce qu'il pense que l'on attend de lui. Les données expérimentales sont alors biaisées (Stengers, 1993, p. 102).

Les animaliers, au contraire, font usage de leur compréhension de l'animal. Leur approche est phénoménologique au sens où ils reconnaissent à l'animal une sphère d'interaction et d'influence et une certaine liberté d'interagir ou non (Merleau-Ponty, 1995) ${ }^{8}$. Pour Merleau-Ponty en effet, l'instinct de l'animal ne peut être ramené à « une mécanique de développement ${ }^{9} »$. Il existe bien pour l'animal, « une marge à l'intérieur de laquelle le choix de l'objet est libre ${ }^{10} »$. Cette façon mouvante d'être au monde pourrait enrichir l'approche analytique adoptée par les chercheurs pour appréhender le bien-être animal.

Les animaliers ont d'abord choisi leur métier parce qu'ils aimaient les animaux. Cela n'en fait pas pour autant des tenants des thèses de Regan (1983), mais peutêtre de celles de Feinberg (1978). Afin d'assurer une protection aux animaux, ces philosophes leur ont attribué des intérêts ou des droits plus ou moins étendus. Selon Joël Feinberg, certains animaux auraient une vie conative - des émotions, des désirs et des états mentauxet, par là même, le droit à la santé, à une liberté de mou-

\footnotetext{
8 Cela renvoie à la notion d'Umwelt de von Uexküll (1934) et à l'interprétation qu'en fait Merleau-Ponty: «aspect du monde en soi auquel l'animal s'adresse, qui existe pour le comportement d'un animal, mais non forcément pour sa conscience », p. 220.

9 Ibid., p. 249.

10 Ibid., p. 252.
} 
vement et à l'intégrité corporelle, mais pas le droit à la vie. En revanche, Tom Regan attribue à certains animaux une valeur intrinsèque (en tant que sujets d'une vie) qui leur confère un droit à la vie. Il en résulte « une obligation directe prima facie de ne pas causer un dommage à ces individus qui ont une expérience propre de leur bienêtre $^{11}$ », quand bien même il en résulterait des conséquences défavorables pour le plus grand nombre (dont les êtres humains).

\section{Trajectoire personnelle et éthique}

L'éthique des animaliers est fondée sur les relations directes qu'ils entretiennent avec les animaux. Mais ces relations sont variables selon les contextes.

Les animaliers de laboratoire ont une éthique utilitariste. Ils disent qu'ils « produisent des souris pour les positionner dans un protocole expérimental ». Les relations qu'ils établissent avec ces rongeurs, nombreux et anonymes, sont caractérisées par un faible engagement affectif et leur assimilation à du matériel biologique. L'acceptation de l'éventuelle souffrance animale dépend alors essentiellement de l'évaluation des bénéfices escomptés pour les humains. C'est pourquoi, ils disent attacher de l'importance à un avis émis par un comité d'éthique.

Malgré ce faible attachement, l'euthanasie semble poser un problème, même lorsqu'elle est pratiquée régulièrement. En effet, ils n'aiment pas en parler et sont plutôt critiques sur les méthodes recommandées par les instances spécialisées.

«Dans l'élevage, s'il y a des souris en trop, j'essaie de les refourguer à quelqu'un plutôt que de les euthanasier sans qu'elles soient mises en expérimentation. Il paraît qu'avec le temps, ça passe, on s'habitue. » (Animalière rongeurs.)

«Les 3/4 des chercheurs n'aiment pas faire les dislocations cervicales (des souris). Certains me demandent, mais je n'aime pas trop. C'est un service assez dur... Je préfère qu'ils me demandent s'ils ne savent pas, plutôt que de voir souffrir la souris. » (Animalière rongeurs.)

Ils sont également vigilants au fait que « les chercheurs consomment trop d'animaux » et "en utilisent pour rien ». Les jeunes pensent que ce malaise «passera » avec l'habitude, affirmation qui reste cependant à démontrer. "Si quelqu'un a fait un mauvais protocole, les résultats partent à la poubelle et les souris sont mortes pour rien... Ça engage ta responsabilité par rapport à un être vivant qui ne peut pas se défendre. » (Animalière rongeurs.)

Chez les animaliers, le critère de l'espèce animale ne tient pas uniquement à des éléments symboliques qui auraient tendance à avantager certains animaux familiers. Il s'agit d'éléments plus concrets tels que la taille de l'animal, sa valeur scientifique et la durée de l'expérimentation, autant de facteurs qui le singularisent aux yeux de

\footnotetext{
11 Op. cit., p. 262.
}

son soigneur. La valeur affective entre également en ligne de compte pour ceux qui ont présidé à sa conception et l'ont aidé à naître, et pour ceux qui lui ont prodigué des soins particuliers. Ainsi, les animaliers ont revendiqué le droit de ne pas participer à l'euthanasie des animaux qu'ils ont soignés (droit de réserve). «Par exemple, S, elle s'occupe de l'élevage, elle les voit grandir. Elle ne peut pas les tuer, on le fait à sa place. » (Animalière responsable d'expériences.)

Les animaliers "gros animaux » sont culturellement liés à leurs animaux par un «contrat domestique » qui leur impose de les protéger et d'en prendre soin pendant toute la durée de leur vie dans l'exploitation agricole en échange de leur production (Larrère et Larrère, 2000). Ce contrat implique également une forme de communication, « un échange d'affect, d'émotion et d'information interprétable par chaque partie », ce qui influencerait nécessairement toute évaluation éthique.

"Quand on est éleveur, il faut savoir que, malheureusement, ça ne va pas toujours comme on voudrait... Avec les bêtes, on a quelquefois des soucis. [... ] Il faut aimer les animaux en sachant que c'est de l'expérimentation, si on veut avancer, on est aussi conduits à faire quelques sacrifices, malheureusement. .. sur les animaux. » (Animalier «petits ruminants ».)

«Depuis toute petite, j'ai voulu travailler avec des animaux... et je pense que c'est une passion avant tout. » (Animalière « petits ruminants ${ }^{12}$.)

Dans l'unité expérimentale «gros animaux » du centre de Jouy, le nombre d'animaux (300) et le rythme de travail sont proches de ceux d'une exploitation traditionnelle. Les éleveurs peuvent communiquer avec les animaux, les connaître individuellement et ils savent quand ils sont en mauvaise santé. Leur vocabulaire exprime le travail du soigneur. Ils disent qu'ils « font naître des veaux et les suivent ».

Toutefois, les conditions d'élevage dans une ferme expérimentale entraînent des situations inédites, qui mettent en péril le contrat "domestique » décrit plus haut. Ainsi, les vaches laitières produisent du lait qui est interdit à la consommation. Par conséquent, leur présence dans le troupeau n'est justifiée que par leur intégration dans un protocole expérimental. Dans le cas contraire, les éleveurs se trouvent devant un paradoxe : celui de prendre soin d'animaux inutiles tant d'un point de vue de la production alimentaire que de la recherche. La seule réponse possible à l'heure actuelle est l'euthanasie de l'animal. Or, comme le disent les animaliers, il s'agit de ne pas «gaspiller une vie». «On est avant tout éleveur et après expérimentateur. À chaque expérience qu'on met en route, il faut un animal en bonne santé, en bonne condition pour un résultat fiable. » (Animalier bovin $^{13}$.)

\footnotetext{
12 Extraits de Paroles d'animaliers.

13 Extrait de Paroles d'animaliers.
} 
Cette attitude est encore plus prégnante lorsque les animaux ont fait l'objet de soins spécifiques et lorsqu'ils sont singuliers, comme les animaux clonés et transgéniques (de Montera, 2007). Les animaliers peuvent alors vivre l'euthanasie comme un échec lorsqu'elle ne conduit pas à la production de résultats expérimentaux. Certaines questions émergentes justifient la mise en place d'un processus décisionnel collectif rassemblant animaliers et chercheurs. Ce fut le cas pour décider l'arrêt des gestations problématiques pour une vache porteuse de veaux clonés hypertrophiés ${ }^{14}$.

L'épreuve de l'expérimentation vécue par les animaliers les met face à la double responsabilité de veiller au bien-être des animaux et de mener à bien le protocole expérimental, même si ce dernier va à l'encontre de l'intérêt des animaux. Nous avons pu constater qu'ils balancent entre une éthique utilitariste et une éthique du soin qui vise avant tout à soulager les souffrances. Proche de l'éthique des vétérinaires ou de l'éthique de la médecine hippocratique visant à agir pour soulager au moment opportun ${ }^{15}$, cette éthique du soin demeure le plus souvent implicite dans les collectifs de recherche où l'expression de l'inquiétude à l'égard des animaux est souvent occultée (Porcher, 2002).

\section{Les animaliers, garants de l'éthique et de la qualité en recherche}

$C^{\prime}$ est la mise en lumière de la relation aux animaux et de la responsabilité morale qu'elle induit qui fait des animaliers les garants de l'éthique en expérimentation animale. De surcroît, leur vision de la science les rend plus proches du citoyen que les scientifiques. Cette divergence de vue sur la finalité de la science a déjà été évoquée par Max Weber (1963, pp. 88 et 90), selon qui «l'homme de science » (le chercheur) s'occuperait plutôt de la science "pour la science même" dans l'espoir de rendre le cours de la vie maîtrisable et prévisible par la connaissance. En revanche, "l'homme de la pratique » (celui qui réalise les protocoles) trouve un sens à ce qu'il fait dans une finalité technique de la science. Il a besoin de croire aux perspectives de progrès qu'elle offre et donc de connaître les bénéfices attendus (et finalement obtenus). Cependant, les animaliers n'acceptent pas l'instrumentalisation totale des animaux au profit du progrès. Or, toujours selon Max Weber, le progrès conduit fatalement à l'instrumentalisation de tout ce qui nous entoure,

\footnotetext{
${ }^{14}$ Conflit de valeurs pour savoir qui avantager du veau cloné qui a une grande valeur scientifique et financière, ou de sa mère porteuse qui souffre.

15 «Ne pas augmenter le mal, mais l'épuiser en administrant ce qui est le plus hostile à chaque maladie [... ]. [.. . ] à condition de discerner les façons opportunes [kairous] d'administrer les choses utiles» (Hippocrate, La Maladie sacrée).
}

y compris les animaux, parce qu'il passe par une intellectualisation toujours croissante. Cette instrumentalisation a pour effet de gommer le côté justement fluctuant et imprévisible de la vie et mène au «désenchantement du monde ».

En 2008, les ministères de la Recherche et de 1'Enseignement Supérieur d'une part, et de l'Agriculture et de la Pêche d'autre part, ont publié une "charte nationale portant sur l'éthique en expérimentale animale » qui rend obligatoire la soumission des protocoles expérimentaux à un comité local d'éthique. Pour que leur protocole soit réalisé dans une animalerie, les chercheurs auront obligation de présenter l'avis du comité d'éthique concerné au $1^{\mathrm{er}}$ janvier 2013 (directive 2010/63/EU). Cet avis est émis après examen du niveau de douleur infligé, du nombre d'animaux utilisés et du bien-fondé de l'utilisation de ce protocole au regard de la littérature scientifique existante dans le but de répondre à la question posée.

En expérimentation animale, chaque situation est unique et il $\mathrm{n}^{\prime} \mathrm{y}$ a pas de règles de déontologie pour chaque cas, mais en revanche, des savoir-faire et des valeurs éthiques partagés par le personnel au contact des animaux. En tant qu'experts seuls capables d'identifier des modifications de comportement indicatrices d'une dégradation de l'état de santé des animaux, les animaliers sont garants de la qualité de la recherche, et, donc, de la fiabilité des mesures et des observations. De plus, leur comportement a une influence sur le bien-être des animaux et, par là même, sur la qualité des résultats (Hemsworth, 2003). Ils assument pleinement cette responsabilité et il n'est pas rare qu'ils « repassent le weekend pour s'assurer que tout va bien ", parfois même en dehors des astreintes qu'ils doivent assurer.

\begin{abstract}
«De toute façon, quand l'animal souffre, les résultats des manips sont nuls. Ça ne marche pas. » (Technicien animalier porcs, proche de la retraite.)

«J'ai tout un volet de ma thèse [... ] sur des ovocytes récupérés en fin de lactation. Donc, toute la partie expérimentale est réalisée à B. [...] Ma collaboration avec les animaliers de $\mathrm{B}$. consiste, du début à la fin de mon protocole, [...] à nourrir les vaches, faire en sorte qu'elles puissent ovuler, récolter les ovocytes et après les trier [... ] c'est toute la récolte de mon matériel biologique. Toute la base de mon travail repose sur leur travail. » (Doctorante ${ }^{16}$.)
\end{abstract}

Ce rôle majeur implique que l'expertise des animaliers soit reconnue et leur jugement respecté. Or cette expertise, constituée principalement de connaissances tacites, n'est pas facilement admise dans un monde professionnel caractérisé par une rationalité scientifique. Une récente étude historique a montré que la reconnaissance de l'expertise des "garçons de laboratoire » employés par les universités anglaises vers 1950 s'était heurtée aux mêmes difficultés (Tansey, 2008).

16 Extrait de Paroles d'animaliers 


\section{Une nécessaire discussion éthique entre les acteurs}

\section{Pour une évaluation éthique indépendante}

Dans cette nouvelle configuration, il sera sans doute nécessaire de clarifier les rôles et responsabilités de chacun et d'inventer des espaces de débat sur des questions éthiques. La charte nationale portant sur l'éthique en expérimentation animale et la directive 2010/63/EU devraient y contribuer. Nous attendons avec impatience le salutaire appui que devraient représenter les comités locaux d'éthique. Les animaliers avouent que l'avis rendu par un tel comité représente une garantie du sens que revêtent les expérimentations animales programmées. Au contraire, un chercheur sûr de son jugement personnel (et qui ne participe pas physiquement aux expérimentations animales) entrevoit la saisine d'un comité d'éthique comme une tracasserie administrative.

Nous avons mis en place des espaces d'échange où des questions pratiques liées à l'éthique ont été débattues entre animaliers et chercheurs : limiter les commandes inutiles d'animaux, améliorer les conditions d'euthanasie, définir un point limite acceptable, informer les animaliers et leur demander leur avis sur la légitimité des protocoles... Nous sommes persuadées que seuls ces échanges, aujourd'hui souvent insuffisants, peuvent permettre de trouver une solution qui tienne compte des valeurs éthiques de chacun. Il est indispensable de permettre l'expression de valeurs éthiques liées au statut de la connaissance et au statut des animaux, et de prendre en compte de manière lucide et responsable le besoin qui existe encore actuellement d'utiliser des animaux en recherche.

\section{Organisation de la recherche, communication et éthique}

Nous avons déjà pointé le fait que l'animal est d'autant plus facilement instrumentalisé que l'on en est éloigné. La distance physique entre chercheurs et animaux est favorisée par l'évolution de la recherche telle que nous l'observons aujourd'hui. Il existe, d'une part, une organisation de plus en plus cloisonnée, avec des prestataires de service spécialisés en expérimentation animale, et, d'autre part, une complexité grandissante de la biologie qui conduit des chercheurs de diverses disciplines (biologistes moléculaires, bio-informaticiens, microbiologistes...) à utiliser, voire à faire produire, des échantillons ou des données issus de l'expérimentation animale sans jamais entrer en relation avec les animaux. Or il est possible que ces chercheurs n'aient reçu aucune formation en physiologie au cours de leur cursus. Par conséquent, il nous semble crucial que ceux qui œuvrent dans ces domaines reçoivent une formation pratique, validée par des stages, auprès des animaliers spécialistes des espèces concernées.

Il nous paraît indispensable de favoriser le développement d'une véritable recherche où la connaissance pratique informelle des animaliers pourrait être exploitée conjointement avec les observations des vétérinaires et des éthologues pour définir des indicateurs fiables de la douleur et du bien-être animal, tant objectifs, par la mesure de paramètres biologiques, que subjectifs, par l'étude des comportements (Hawkins, 2002). Les modes de communication explorés dans les collectifs de recherche participative, en particulier ceux formés avec les agriculteurs (Hoffman et al., 2007), pourraient être utilisés. De la même façon, les chercheurs contribueraient à expliciter les connaissances tacites accumulées par les animaliers sur le terrain.

Enfin, l'appartenance des animaliers à la communauté scientifique doit être reconnue par le partage de codes qui lui sont propres, dont le principal est la publication scientifique. Même s'ils n'ont pas leur place en tant qu'auteurs, leur mention explicite dans les remerciements leur procurera une reconnaissance plus étendue, puisqu'à l'avenir, elle devrait être prise en compte par les systèmes internationaux de référencement des publications scientifiques ${ }^{17}$.

\section{Conclusions}

En conclusion, cet article avait pour objectif de diffuser les résultats de l'expérience de mise en mots que nous conduisons depuis plus de quatre ans autour de l'expérimentation animale dans un centre de recherche de l'Inra. Cette expérience a montré que, contrairement à ce que pourrait laisser croire l'éthique réglementaire, le positionnement éthique des acteurs n'était pas consensuel, et qu'il dépendait pour beaucoup de la relation directe qu'ils construisent avec l'animal d'expérience. Nous avons mis en lumière les écarts entre une éthique «officielle» (utilitarisme pathocentrique) et les éthiques implicites des membres du collectif. À une reconnaissance "molle » d'une sensibilité des animaux par ceux qui s'en tiennent à distance s'oppose une réelle responsabilité éthique de ceux qui entrent en relation directe avec eux.

Il nous semble indispensable de placer les animaliers au centre du dispositif de recherche, car ils jouent un rôle de médiation entre la sphère géographique, relationnelle et sémantique incluant les animaux, et la sphère des concepteurs. Ce rôle essentiel doit être reconnu et mener à une généralisation de la délibération éthique entre membres d'un collectif chaque fois qu'émergent

\footnotetext{
17 À cet égard, il faut saluer la remise par l'Inra d'une distinction, les lauriers, commune à toutes les catégories de personnel.
} 
des questions complexes ou nouvelles soulevées par les biotechnologies. De plus, l'expérimentation animale doit être considérée dans une approche « $3 R$ » qui intègre les méthodes alternatives dans une démarche globale. Ainsi, chacun des acteurs du collectif de recherche pourrait, avec le recul nécessaire, tenter de rendre compte des enjeux de l'expérimentation animale auprès du grand public. Il s'agit d'un réel changement des mentalités qui nécessitera des efforts de formation et de communication interne.

\section{Remerciements}

Nous remercions chaleureusement ceux et celles qui ont contribué à la réalisation de ce travail. Les témoignages des animaliers et des chercheurs en constituent le socle. Emmanuel Jolivet, président du centre de recherche, a constamment soutenu cette démarche qu'il a largement contribué à initier, et pour laquelle il nous a prodigué encouragements et conseils. Enfin, cet article a bénéficié des commentaires de Raphaël Larrère, Olivier Rampin et Jean-Paul Renard.

\section{Références}

Bentham, J., 1789. An Introduction to the Principles of Morals and Legislation, London, T. Payne \& Son (pour l'édition de 1996 : Bentham, J., 1996. An Introduction to the Principles of Morals and Legislation, Oxford, Clarendon Press/New York, Oxford University Press).

Birke, L., Arluke, A., Michael, M., 2007. The Sacrifice: How Scientific Experiments Transform Animals and People, West Lafayette, Purdue University Press.

Canguilhem, G., 1977. Idéologie et rationalité dans l'histoire des sciences de la vie, Paris, Vrin.

de Montera, B., 2000. Vers une éthique naturaliste. Mémoire de Diplôme d'études approfondies en philosophie des sciences, Université Paris 1 Panthéon-Sorbonne, Paris.

de Montera, B., 2007. Le clonage animal : de l'animal de laboratoire, à l'animal de production, à l'animal de compagnie, in Parizeau, M.-H., Chapouthier, G. (Eds), L'Être humain, l'animal et la technique, Québec, Presses de l'Université Laval, 97-119.

Despret, V., Porcher, J., 2007. Être bête, Paris, Actes Sud.

Dockès, A.C., Kling-Eveillard, F., 2007. Les représentations de l'animal et du bien-être animal par les éleveurs français, Productions animales, 20, 1, 23-28.

Feinberg, J., 1978. Human duties and animal rights, in Morris, R.K., Fox, M.W. (Eds), On the Fifth Day: Animal Rights and Human Ethics, Washington, Acropolis Books, 45-69.
Grohs, C., Bernard, J., 2007. Création d'un questionnaire sur l'éthique et l'expérimentation animale au centre INRA de Jouyen-Josas. Rapport de Licence professionnelle de biotechnologies, Université de Versailles-Saint-Quentin, Saint-Quentinen-Yvelines.

Hawkins, P., 2002. Recognizing and assessing pain, suffering and distress in laboratory animals: A survey of current practice in the UK with recommendations. Laboratory Animals, 36, 378-395.

Hemsworth, P.H., 2003. Human-animal interactions in livestock production. Appl. Anim. Behav. Sci., 81, 185-198.

Hobbes, T., 1651 [1 $1^{\text {ère }}$ éd. : 1642]. De Cive, London, R. Royston. Trad. fr. : Le Citoyen ou les fondements de la politique, Paris, Flammarion, 1982.

Hoffmann, V., Probst, K., Christinck, A., 2007. Farmers and researchers: How can collaborative advantages be created in participatory research and technology development? Agriculture and human values, 24, 355-368.

Kant, E., 1924, Eine Vorlesung Kants über Ethik, Berlin, Pan Verlag, R. Heise. Trad. fr. : Leçons d'éthique, Paris, Librairie générale française, 1997.

Larrère, R., 2002. Éthique et expérimentation animale, Natures Sciences Sociétés, 10, 1, 24-32.

Larrère, C., Larrère, R., 2000. Animal rearing as a contract? J. Agric. and Environ. Ethics, 12, 51-58.

Lhoste, E.F., 2003. L'Expérimentation animale face à la communication : la difficulté d'en parler ensemble révélatrice du décalage entre science et société ? Mémoire de Master 2, Université de Versailles-Saint-Quentin, Saint-Quentin-en-Yvelines.

Lhoste, E.F., de Montera, B., Pleinet, S., Rampin, O., 2008. Promouvoir la discussion éthique au sein d'un collectif de recherche : les actions conduites au sein du centre de recherche INRA de Jouy-en-Josas. Communication au $34^{\mathrm{e}}$ colloque de l'Association française des sciences et techniques de l'animal de laboratoire, Strasbourg, 4-6 juin.

Merleau-Ponty, M., 1995. La Nature. Notes. Cours du Collège de France (1956-1960), Paris, Le Seuil.

Porcher, J., 2002. L'occultation de l'affectivité dans l'expérimentation animale : le paradoxe des protocoles, Natures Sciences Sociétés, 10, 1, 33-36.

Regan, T., 1983. The Case for Animal Rights, London, Routledge \& Kegan.

Russell, W.M.S., Burch, R.L., 1959. The Principles of Humane Experimental Technique, London, Methuen.

Singer, P., 1975. Animal Liberation: A New Ethics for our Treatment of Animals, New York, New York Review/Random House. Trad. fr. : La Libération animale, Paris, Grasset, 1993.

Stengers, I., 1993. L'Invention des sciences modernes, Paris, La Découverte.

Tansey, E.M., 2008. Keeping the culture alive: The laboratory technician in mid-twentieth century medical research, Notes and records of The Royal Society, 62, 1, 77-95.

Uexküll, J. von, 1934. Streifzüge durch die Umwelten von Tieren und Menschen, Berlin, J. Springer. Trad. fr. : Mondes animaux et monde humain, Paris, Gonthier, 1956.

Weber, M., 1963. Le Savant et le politique, Paris, Union générale d'éditions. 